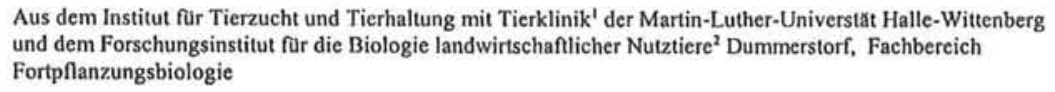
und dem Forschungsinstitut fur die Biologie landwirtschafticher Nutztiere ${ }^{2}$ Dummerstorf, Fachbereich

SVEN BIEREDER', MICHAEL WICKE', GERHARD von LENGERKEN', FALK SCHNEIDER ${ }^{2}$ und WILHELM KANITZ ${ }^{2}$

\title{
Muskelwachstum und IGF-I bei Schweinen unterschiedlichen Geschlechts*
}

\author{
Herrn Professor Dr. Peter Glodek zum 65. Geburtstag gewidmet
}

\begin{abstract}
Summary
Title of the paper: Growth of skeletal muscle and IGF-I in pigs of different sex

IGF-I is a pluripotent factor that is involved in regulation of growth, differentiation and a large number of functions in numerous tissues and their cells. IGF-I is synthesized by hepatocytes (endocrine role) and several extrahepatic tissues (e.g. skeletal muscle; autocrine and paracrine role). In our study, we describe the postnatal growth of the skeletal muscles in pigs of various sex taking into account the possible influence of endogenous IGF-I.

The investigation was made on 42 crossbred pigs. Seven blood samples and 4 biopsy samples of two muscles ( $M$. longissimus dorsi and $M$. triceps brachii) were taken for the determination of IGF-I blood plasma concentration and muscle fibre diameter, respectively as well as for further muscle structural and biochemical traits. IGF-I plasma concentrations show an increase during fattening with significantly highest levels for boars. Phenotypic differences between sows and boars in thickness of shoulder muscle are proven after the day 181 with ultrasonography because significant differences were detected in mean muscle area of caput longum musculi triceps brachii between sows and boars and barrows (180th day of life). There are no significant differences in mean muscle fibre diameter of both muscles between sexes. A group of animals with high mean diameter in muscle fibres (day 200) of $M$. triceps brachii has significantly higher IGF-I concentrations in blood plasma than a group of animals with low muscle fibre diameter in the same muscle.
\end{abstract}

Key Words: pig, IGF-I, M. triceps brachii, M. longissimus dorsi, muscle fibre diameter, muscle area

\section{Zusammenfassung}

Der insulinähnliche Wachstumsfaktor-I gilt als wichtige Komponente der Wachstumsregulation über die Growth Hormone-Achse und ist für anabole Prozesse in einer Vielzahl von Geweben essentiell. Seine Bildung erfolgt hepatisch (endokrine Funktion) und extrahepatisch (z.B. Muskulatur: autokrine und parakrine Funktion). Zum besseren Verständnis seiner Rolle bei der Regulation des Wachstums der Skelettmuskulatur des Schweines wurde eine Untersuchung an 42 Probanden einer Dreirassenkreuzung vom Zeitpunkt der Geburt bis zum 210. Lebenstag durchgeführt. In diesem Zeitraum wurden die Blutplasmakonzentrationen des IGF-I und parallel dazu die Entwicklung der Muskelquerschnittsflächen, der Muskelfaserdurchmesser und Muskelfasertypen an zwei Muskeln untersucht. Die Blutplasmakonzentrationen zeigen signifikante Differenzen zwischen einerseits Ebern und andererseits Sauen und Kastraten. Am 180. Lebenstag zeigen Sauen signifikant kleinere Muskelflächen im Caput longum musculi triceps brachii als Eber und Kastraten. Die mittleren Muskelfaserdurchmesser beider Muskel zeigen bis zum 180. bzw. 200. Lebenstag keine geschlechtsspezifischen Unterschiede. Tiere mit hohen Faserdurchmessern im $M$. triceps brachii (200. Lebenstag) besitzen signifikant höhere IGF-IBlutplasmakonzentrationen als Tiere mit niedrigen Faserdurchmessem im gleichen Muskel.

Schlüsselwörter: Schwein, IGF-I, M. triceps brachii, M. longissimus dorsi, Muskelfaserdurchmesser, Muskelfläche

- gefordert durch die Deutsche Forschungsgemeinschan (DFG) 
1. Einleitung

IGF-I, bei dem es sich um ein basisches Polypeptid von 70 AS und 7646 Da handelt (RINDERKNECHT und HUMBEL, 1978), gilt als postnatal wichtiger Faktor bei der Regulation des Wachstums. Seine durch Somatotropin $(\mathrm{GH})$ gesteuerten Wirkungen zielen in erster Linie auf die Auslösung von Mitosen und die Förderung der Proteinsynthese im Zielgewebe ab. Humaner, boviner und porciner IGF-I sind identisch (HONEGGER und HUMBEL, 1986). IGF-I wird im adulten Organismus vor allem in der Leber produziert und in die Blutbahn sezerniert. Die Serumspiegel schwanken je nach Geschlecht, Alter, Ernährungszustand und Hormonstatus stark. Er wirkt im Blut als klassisches endokrines Agens durch Wechselwirkungen mit spezifischen Zelloberflächenrezeptoren. Ein Großteil des IGF-I liegt in Bindung an eine Reihe von spezifischen Proteinen (IGF-BP) vor. Bisher wurden 7 Vertreter beschrieben, wobei im Plasma die Bindung an das IGF-BP3 überwiegen soll (LITWACK, 1994). Diese Komplexbildung mit Bindungsproteinen dient wahrscheinlich dazu, die Peptide vor dem Abbau zu schützen und deren Halbwertszeit zu verlängern. Eine Vielzahl extrahepatischer Gewebe (z.B. Muskulatur) ist ebenfalls in der Lage, IGF-I zu sezernieren. So wird angenommen, daß diese nichthepatischen IGF-I-Quellen in erster Linie am Ort ihrer Synthese wirken, so an angrenzenden Zellen (parakrine Wirkung) oder an den gleichen Zellen (autokrine Wirkung) die den IGF-I bilden (LITWACK, 1994).

Nachfolgend werden ausgewählte Ergebnisse aus einer Longitudinalstudie zum postnatalen Wachstum der Skelettmuskulatur bei Schweinen unterschiedlichen Geschlechts dargestellt und besonders auf mögliche Wechselwirkungen mit wachstumsbeeinflussenden körpereigenen Hormonen der GH-IGF-Achse am Beispiel des IGF-I eingegangen.

2 . Material und Methode

Die Untersuchungen erfolgten an 42 Probanden der genetischen Konstruktion Pietrain $x$ (Deutsches Edelschwein $x$ Deutsche Landrasse) im Nutztierwissenschaftlichen Zentrum Merbitz der Universität Halle-Wittenberg.

Es wurden Probanden mit annähernd gleichen Geburtsgewichten $(1525 \mathrm{~g} \pm 50 \mathrm{~g})$ ausgewählt. Aus jedem Wurf standen jeweils ein weibliches, ein intaktes männliches Tier sowie ein Kastrat für die Untersuchungen zur Verfügung.

Die Mast erfolgte zeitabhängig vom 28. bis 210. Lebenstag in Einzelhaltung mit Sichtkontakt, das Alleinfuttermittel (Ferkelaufzuchtfutter, Mastfutter I, Mastfutter II) stand ad libitum zur Verfügung.

Im Versuchsverlauf wurden insgesamt 7 Blutproben pro Tier jeweils zum gleichen Zeitpunkt entnommen. Die Hormonanalysen erfolgten im Forschungsinstitut für die Biologie landwirtschaftlicher Nutztiere Dummerstorf, Forschungsbereich Fortpflanzungsbiologie. Dabei wurde ein ${ }^{125}$ I-RIA (Nichols Institute, Bad Nauheim) nach vorangegangener Säure-Ethanol-Fällung zur Trennung des IGF-I von begleitenden Bindungsproteinen eingesetzt. Die Entnahmen der Muskelproben am lebenden Tier erfolgten am $M$. longissimus dorsi in Höhe der zweit- / drittletzten Rippe etwa $5 \mathrm{~cm}$ lateral der Medianebene mittels Schußbiopsie bzw. am M. triceps brachii an der dicksten 
Stelle des Caput longum unter Ultraschallkontrolle mittels einer Quick-Core Biopsiekanüle der Firma Cook. Nach Aufblocken auf Kork mittels Zelleim und Talkum wurden die Muskelproben in flüssigem Stickstoff gelagert. Parallel dazu wurden Messungen der Muskelfächenmaße des $M$. longissimus dorsi und des Caput longum des $M$. triceps brachii mit einem Ultraschallgerät ALOKA SSD 500 mit den Schallköpfen UST-5039-P und UST-5011U-3.5 auf Hohe der genannten Biopsiestellen durchgefuhrt.

Die Bestimmung der mikrostrukturellen Merkmale (Fasertyp, Fasertypenanteile, Faserdurchmesser, Faserfläche) erfolgte anhand von $10 \mu \mathrm{m}$ dicken Gefrierschnitten, die mit Hilfe eines Cryocut Jung CM 1800 der Firma Leica hergestellt wurden und folgender kombinierter ATPase-Färbung nach HORAK mittels eines halbautomatischen Bildanalysesystems (Lucia M).

Die Datenauswertung erfolgte mit den Programmen SAS Version 6.10 (SAS Inst. Inc. 1989) und STATISTICA for Windows (Vers. 5.1 StatSoft Inc. 1996) mittels Varianzanalyse nach folgendem Grundmodell:

$$
\begin{array}{rll}
\mathrm{Y}_{\mathrm{ijk}} & =\mu+\mathrm{SEX}_{\mathrm{i}}+\mathrm{V}_{\mathrm{j}}+\mathrm{M}_{\mathrm{k}}+\mathrm{e}_{\mathrm{ijk}} \\
\mathrm{Y}_{\mathrm{ijk}} & =\quad \text { Beobachtungswert des Einzeltieres } \\
\mu & =\quad \text { Mittelwert des Merkmals } \\
\mathrm{SEX}_{\mathrm{i}} & = & \text { fixer Effekt des i-ten Geschlechts }(\mathrm{i}=1,2,3) \\
\mathrm{V}_{\mathrm{j}} & = & \text { fixer Effekt des } \mathrm{j} \text {-ten Vaters }(\mathrm{j}=1,2) \\
\mathrm{M}_{\mathrm{k}} & = & \text { fixer Effekt der k-ten Mutter }(\mathrm{k}=1 \ldots 16) \\
\mathrm{e}_{\mathrm{ijk}} & = & \text { Restfehler }
\end{array}
$$

Signifikante Unterschiede ( $\mathrm{p} \leq 0,05)$ sind durch unterschiedliche Buchstaben gekennzeichnet.

\section{Ergebnisse}

Bei Betrachtung ausgewählter Parameter der Mast-und Schlachtleistung der Tiere zeigen die Eber erwartungsgemäß die signifikant höchsten Lebenstagszunahmen (LTZ) im Prüfzeitraum (Tag 28-210), gefolgt von Kastraten und Sauen (Tab. 1). Damit gekoppelt ist der bei Ebern signifikant niedrigere Futteraufwand (FuA) im Vergleich zu Kastraten und Sauen als Ausdruck ihres starken anabolen Potentials.

Die Schlachtkörpergewichte (SGWw) der Eber sind demzufolge am höchsten und unterscheiden sich signifikant von denen der Sauen. Für Sauen und Eber ist im Vergleich zu den Kastraten ein signifikant höherer Fleischanteil nachweisbar.

Die mittels Ultraschall ermittelten Flächenmaße des $M$. longissimus dorsi (in Höhe 2./3.-letzte Rippe) haben im Zeitraum vom 01.-180. Lebenstag keine sigifikanten Unterschiede im Geschlechtervergleich (Tab. 2). Der Wachstumsverlauf ähnelt einer sigmoidalen Kurve, der stärkste Anstieg erfolgt bei Ebern später (etwa bei $80-100 \mathrm{~kg}$ ) als bei Kastraten und Sauen $(60-80 \mathrm{~kg})$. 
Tabelle 1

Least Square-Means (LSM) und Standardfehler (SE) ausgewählter Parameter der Mast- und Schlachtleistung in Abhängigkeit vom Geschlecht (Least square means and standard error of fattening performances and carcass quality depending on sex)

\begin{tabular}{lcccccc}
\hline & \multicolumn{2}{c}{ Kastraten } & \multicolumn{2}{c}{ Eber } & \multicolumn{2}{c}{ Sauen } \\
& LSM & SE & LSM & SE & LSM & SE \\
\hline LTZ (g/d) & $656 \mathrm{a}$ & 15 & $719 \mathrm{~b}$ & 14 & $613 \mathrm{c}$ & 14 \\
FuA (kg/kg) & $3,42 \mathrm{a}$ & 0,06 & $2,97 \mathrm{~b}$ & 0,06 & $3,27 \mathrm{a}$ & 0,06 \\
SGWw (kg) & $110,1 \mathrm{a}$ & 2,51 & $116,2 \mathrm{a}$ & 2,29 & $103,3 \mathrm{~b}$ & 2,37 \\
MFA (\%) & $53,1 \mathrm{a}$ & 0,62 & $54,5 \mathrm{~b}$ & 0,56 & $55,9 \mathrm{~b}$ & 0,58 \\
\hline
\end{tabular}

\section{Tabelle 2}

Least Square-Means (LSM) und Standardfehler (SE) der Muskelflächen $\left(\mathrm{cm}^{2}\right)$ des $M$. longissimus in Abhängigkeit von Alter und Geschlecht (Least square means and standard error of muscle area of $M$. longissimus dorsi depending on age and sex)

\begin{tabular}{ccccccc}
\hline $\begin{array}{c}\text { Alter } \\
\text { in d }\end{array}$ & LSM & SE & LSM & SE & LSM & SE \\
\hline 1 & $1,60 \mathrm{a}$ & 0,06 & $1,64 \mathrm{a}$ & 0,06 & $1,61 \mathrm{a}$ & 0,06 \\
32 & $6,09 \mathrm{a}$ & 0,43 & $6,93 \mathrm{a}$ & 0,40 & $6,48 \mathrm{a}$ & 0,40 \\
78 & $15,29 \mathrm{a}$ & 0,70 & $15,40 \mathrm{a}$ & 0,70 & $15,90 \mathrm{a}$ & 0,70 \\
151 & $40,40 \mathrm{a}$ & 1,18 & $38,10 \mathrm{a}$ & 1,10 & $40,10 \mathrm{a}$ & 1,10 \\
180 & $46,90 \mathrm{a}$ & 0,90 & $46,30 \mathrm{a}$ & 0,80 & $45,00 \mathrm{a}$ & 0,90 \\
\hline
\end{tabular}

Ähnlich stellt sich der Wachstumsverlauf im M. triceps brachii dar. Erst gegen Ende der Prüfperiode (nach dem 180. Lebenstag) haben Sauen signifikant kleinere Flächen als Eber und Kastraten (Tab. 3). Die Eber weisen tendenziell das größte Flächenmaß in diesem Muskel auf. Der Anstieg des Flächenwachstums im M. triceps brachii ist geringer als im $M$. longissimus dorsi.

Tabelle 3

Least Square-Means (LSM) und Standardfehler (SE) der Muskelflächen $\left(\mathrm{cm}^{2}\right)$ des $M$. triceps brachii in Abhängigkeit von Alter und Geschlecht (Least square means and standard error of $M$. triceps brachii muscle area depending on age and sex)

\begin{tabular}{ccccccc}
\hline $\begin{array}{c}\text { Alter } \\
\text { in d }\end{array}$ & LSM & SE & LSM & SE & LSM & SE \\
\hline 32 & $6,93 \mathrm{a}$ & 0,33 & $6,93 \mathrm{a}$ & 0,30 & $6,95 \mathrm{a}$ & 0,32 \\
78 & $16,28 \mathrm{a}$ & 0,95 & $15,91 \mathrm{a}$ & 0,82 & $15,40 \mathrm{a}$ & 0,81 \\
151 & $28,36 \mathrm{a}$ & 0,90 & $26,32 \mathrm{a}$ & 0,82 & $27,12 \mathrm{a}$ & 0,85 \\
180 & $34,82 \mathrm{a}$ & 0,75 & $35,19 \mathrm{a}$ & 0,78 & $31,88 \mathrm{~b}$ & 0,74 \\
\hline
\end{tabular}

Die am lebenden Tier gewonnenen und mittels des halbautomatischen Bildanalysesystems Lucia M erfaßten und ausgewerteten durchschnittlichen Muskelfaserdurchmesser des $M$. longissimus dorsi ergeben im vorliegenden Tiermaterial keine signifikanten Unterschiede im Geschlechtervergleich (Tab. 4). Tendenziell weisen in diesem Versuch die Eber am 200. Lebenstag die größten Faserdurchmesser auf. 
Tabelle 4

Least Square-Means (LSM) und Standardfehler (SE) der Muskelfaserdurchmesser $(\mu \mathrm{m})$ des $M$. longissimus dorsi in Abhängigkeit von Alter und Geschlecht (Least square means and standard error of muscle fibre diameter of $M$. longissimus dorsi depending on age and sex)

\begin{tabular}{ccccccc}
\hline $\begin{array}{c}\text { Alter } \\
\text { ind }\end{array}$ & \multicolumn{2}{c}{ Kastraten } & \multicolumn{2}{c}{ Eber } & \multicolumn{2}{c}{ Sauen } \\
\hline 78 & LSM & SE & LSM & SE & LSM & SE \\
151 & $62,7 \mathrm{a}$ & 1,6 & $60,3 \mathrm{a}$ & 1,4 & 63,1 & 1,4 \\
180 & $93,7 \mathrm{a}$ & 2,5 & $90,7 \mathrm{a}$ & 2,3 & 91,4 & 2,2 \\
200 & $101,8 \mathrm{a}$ & 2,6 & $95,8 \mathrm{a}$ & 2,5 & 100,0 & 2,5 \\
& $106,4 \mathrm{a}$ & 2,3 & $107,9 \mathrm{a}$ & 2,2 & 104,8 & 2,3 \\
\hline
\end{tabular}

Im M. triceps brachii kann - analog zum M. longissimus dorsi und entgegen der Erwartung - kein signifikanter Unterschied der Muskelfaserdurchmesser zwischen den einzelnen Geschlechtern festgestellt werden (Tab. 5). Tendenziell findet man die größten Faserdurchmesser bei Kastraten.

Tabelle 5

Least Square-Means (LSM) und Standardfehler (SE) der Muskelfaserdurchmesser $(\mu \mathrm{m})$ des $M$. triceps brachii in Abhängigkeit von Alter und Geschlecht (Least square means and standard error of muscle fibre diameter of $M$. triceps brachii depending on age and sex)

\begin{tabular}{ccccccc}
\hline $\begin{array}{c}\text { Alter } \\
\text { in d }\end{array}$ & LSM & SE & LSM & SE & LSM & SE \\
\hline 32 & $41,5 \mathrm{a}$ & 1,1 & $41,6 \mathrm{a}$ & 1,8 & $41,0 \mathrm{a}$ & 1,3 \\
78 & $59,5 \mathrm{a}$ & 2,4 & $66,1 \mathrm{~b}$ & 2,1 & $70,5 \mathrm{~b}$ & 2,2 \\
151 & $93,2 \mathrm{a}$ & 3,9 & $90,0 \mathrm{a}$ & 3,9 & $88,6 \mathrm{a}$ & 3,5 \\
180 & $97,8 \mathrm{a}$ & 3,4 & $93,5 \mathrm{a}$ & 3,1 & $91,5 \mathrm{a}$ & 3,1 \\
\hline
\end{tabular}

Die IGF-I-Blutplasmakonzentrationen (Tab. 6) zeigen den erwarteten Anstieg in der Wachstumsperiode. Die höchsten Werten können für Eber und Sauen um den 150. Lebenstag (Phase des stärksten Wachstums, 80-100 kg Körpermasse) und für Kastraten um den 111. Lebenstag (Phase des stärksten Wachstums, Körpermasse etwa 60 kg).

Tabelle 6

Least Square-Means (LSM) und Standardfehler (SE) der IGF-I-Blutplasmakonzentrationen (ng/ml) in Abhängigkeit von Alter und Geschlecht (Least square means and standard error of IGF-I-plasma concentrations depending on age and sex)

\begin{tabular}{ccccccc}
\hline $\begin{array}{c}\text { Alter } \\
\text { in d }\end{array}$ & LSM & SE & LSM & SE & LSM & SE \\
\hline $1 \mathrm{~d}$ & $39,5 \mathrm{a}$ & 2,9 & $36,6 \mathrm{a}$ & 2,5 & $39,5 \mathrm{a}$ & 2,6 \\
$32 \mathrm{~d}$ & $119,7 \mathrm{a}$ & 8,1 & $115,2 \mathrm{a}$ & 7,5 & $131,1 \mathrm{a}$ & 7,2 \\
$78 \mathrm{~d}$ & $161,4 \mathrm{a}$ & 28,0 & $179,8 \mathrm{a}$ & 26,2 & $190,5 \mathrm{a}$ & 29,1 \\
$111 \mathrm{~d}$ & $226,6 \mathrm{ab}$ & 29,4 & $293,0 \mathrm{a}$ & 26,7 & $195,2 \mathrm{~b}$ & 28,1 \\
$151 \mathrm{~d}$ & $169,6 \mathrm{a}$ & 18,9 & $394,5 \mathrm{~b}$ & 17,2 & $211,9 \mathrm{a}$ & 17,9 \\
$180 \mathrm{~d}$ & $142,3 \mathrm{a}$ & 16,0 & $374,2 \mathrm{~b}$ & 14,6 & $202,5 \mathrm{c}$ & 15,1 \\
$200 \mathrm{~d}$ & $130,3 \mathrm{a}$ & 22,3 & $379,1 \mathrm{~b}$ & 19,5 & $184,4 \mathrm{a}$ & 20,1 \\
\hline
\end{tabular}

Bei Sauen kann anhand der vorliegenden Daten kein echtes Maximum ermittelt werden. Ab dem 111. Lebenstag sind die Unterschiede zwischen Ebern und Sauen signifi- 
kant. Am 180. Lebenstag können zwischen Ebern, Sauen und Kastraten signifikante Unterschiede ermittelt werden. Insgesamt pegeln sich die IGF-I-Blutplasmakonzentrationen bei einem bestimmten Niveau ein, bei Sauen und Kastraten mit leicht fallender Tendenz. Eber dagegen bleiben auf hohem Niveau stabil.

Zwischen den IGF-I-Blutplasmakonzentrationen und der Lebenstagzunahme konnten die erwarteten mittleren positiven Zusammenhänge ebenso wie zwischen den IGF-IBlutplasmakonzentrationen und der Lebendmasseentwicklung bestimmt werden. Ferner wurden die erwarteten negative Zusammenhänge zwischen IGF-I-Blutplasmakonzentrationen und dem Futteraufwand (Tab. 7) bestätigt.

Tabelle 7

Korrelationen zwischen IGF-I-Blutplasmakonzentrationen und ausgewåhlten Parametern der Mastleistung (Correlations between IGF-I- plasma concentrations and fattening peformances)

\begin{tabular}{cccc}
\hline & Lebendmasse & FuA (kg/kg) & LTZ (g/d) \\
\hline IGF-I 01. LT & 0,04 & 0,11 & 0,05 \\
IGF-I 32. LT & 0,19 & 0,22 & 0,26 \\
IGF-I 77. LT & $\mathbf{0 , 5 7}$ & $-0,10$ & $\mathbf{0 , 3 2}$ \\
IGF-I 110. LT & $\mathbf{0 , 4 5}$ & $-0,09$ & $\mathbf{0 , 3 2}$ \\
IGF-I 151. LT & 0,22 & $-\mathbf{0 , 4 2}$ & $\mathbf{0 , 4 4}$ \\
IGF-I 181. LT & 0,31 & $-\mathbf{0 , 4 4}$ & $\mathbf{0 , 4 7}$ \\
IGF-I 200. LT & $\mathbf{0 , 3 7}$ & $\mathbf{- 0 , 4 6}$ & $\mathbf{0 , 4 7}$ \\
\hline Markierte Korr. signifikant fur p s0,05 & & &
\end{tabular}

Zur Darstellung möglicher Wechselwirkungen zwischen den Muskelfaserdurchmessern und den Blutplasmakonzentrationen des IGF-I im Wachstumsverlauf wurde eine Gruppeneinteilung der Probanden anhand der Faserdurchmesser im jeweiligen Muskel vorgenommen. Dabei haben Probanden mit einem durchschnittlichen Faserdurchmesser von $\geq 94 \mu \mathrm{m}$ im Caput longum musculi triceps brachii am 200. d höhere IGF-IWerte als Probanden mit einem geringeren Faserdurchmesser. Der Gruppe mit den höheren Faserdurchmessern gehören zu 45\% männliche Tiere an, weibliche und ka-

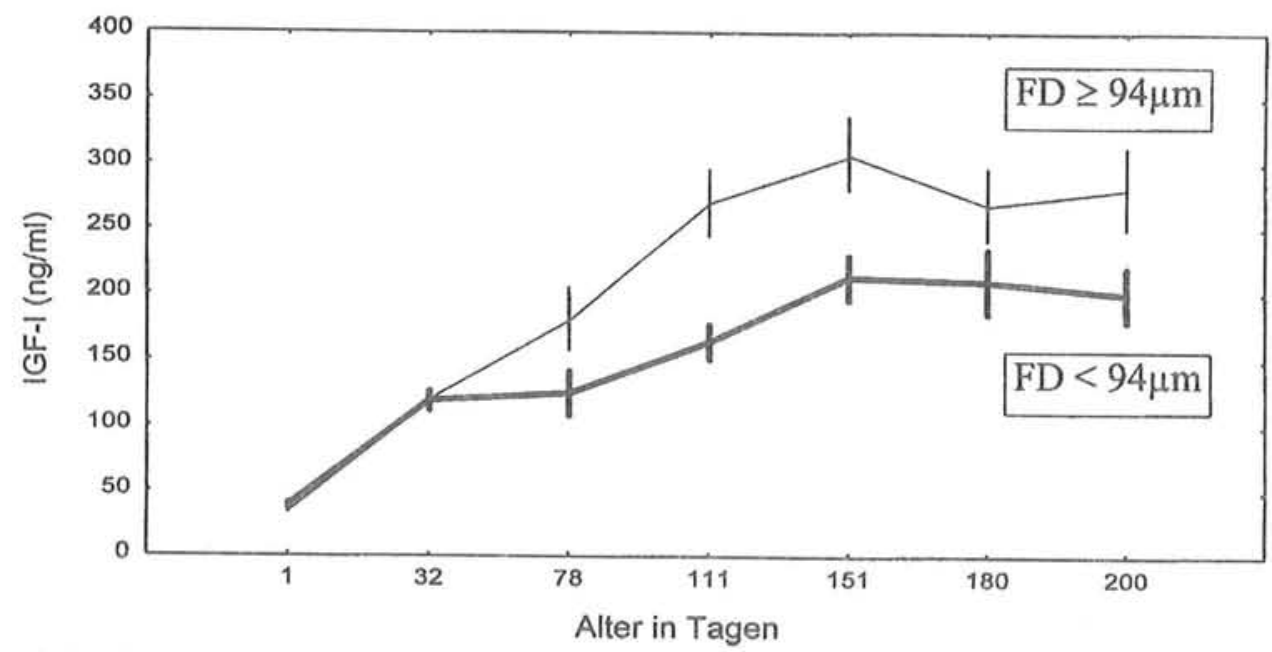

Abb. 1: IGF-I Konzentration im Blutplasma von Schweinen bei Einteilung in Gruppen nach dem Faserdurchmesser (FD) des $M$. triceps brachii (IGF-I plasma concentration of pigs by classification in groups of fibre diameter in $M$. triceps brachii) 
strierte Tiere beteiligen sich zu etwa gleichen Teilen an den restlichen $55 \%$. Im Abschnitt vom 111. bis zum 180. Lebenstag sind diese Unterschiede signifikant (Abb. 1). Auch im $M$. longissimus zeigen Probanden mit großem Faserdurchmesser $(\geq 106 \mu \mathrm{m})$ tendenziell höhere IGF-I-Blutplasmakonzentrationen als Probanden mit einem geringeren Faserdurchmesser. Die Unterschiede zwischen den Gruppen sind geringer als im M. longissimus und können statistisch nicht gesichert werden (Abb. 2).

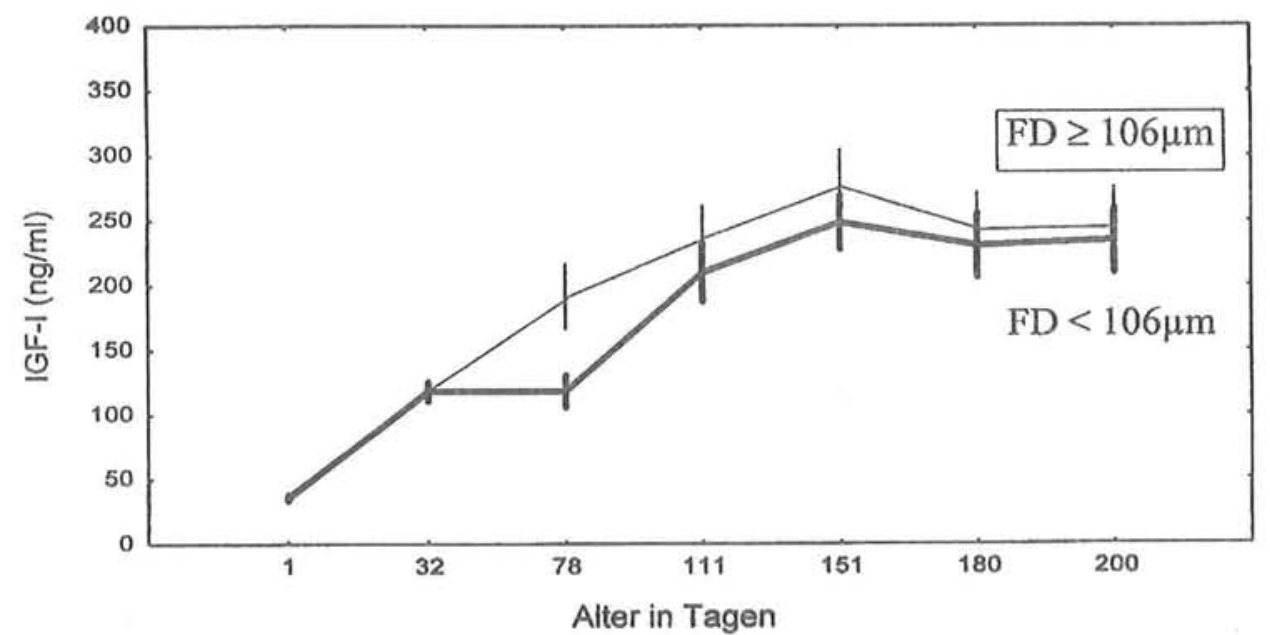

Abb. 2: IGF-I Konzentration im Blutplasma von Schweinen bei Einteilung in Gruppen nach dem Faserdurchmesser (FD) des $M$. longissimus dorsi (IGF-I plasma concentration of pigs by classification in groups of fibre diameter in $M$. longissimus dorsi)

4. Diskussion

Der anabole Effekt der Geschlechtssteroide erklärt die Geschlechtsunterschiede der Masteigenschaften und Schlachtkörpercharakteristika des Schweines. Dabei existiert eine klare Reihenfolge in der Futterverwertung, im Magerfleischanteil und im Fettanteil. Eber sind Sauen in diesen Parametern überlegen, welche wiederum den Börgen überlegen sind (SATHER et al., 1991). Im vorliegenden Versuch zeigt sich auch genau dieses Ergebnis, wenngleich der Magerfleischanteil der Sauen als relativ hoch bewertet werden muß und sich von dem der Eber nicht signifikant unterscheidet. Die Tatsache, daß die Muskelflächenmaße des M. longissimus dorsi der Tiere im Versuch bis zum 180. Lebenstag zwischen den Geschlechtern keinerlei Unterschiede und die Muskelflächenmaße des Caput longum musculi triceps brachii erst ab dem 181. Lebenstag signifikante Unterschiede zwischen einerseits Ebern und Kastraten und andererseits Sauen aufweisen, deutet auf eine geschlechtsdimorphistische Ausprägung der Schultermuskulatur des Schweines -wie äußerlich sichtbar- erst jenseits der Pubertät hin. Möglicherweise sind die Unterschiede an anderen, allerdings sonographisch kaum abzugrenzenden Muskeln des Schulterbereiches (z.B. M. trapezius) deutlicher. Anhand des vorliegenden Datenmaterials können an beiden untersuchten Muskeln keine signifikanten Unterschiede im Muskelfaserdurchmesser zwischen den Ge- 
schlechtern festgestellt werden. Im $M$. longissimus dorsi allerdings weisen die Eber tendenziell die größten Faserdurchmesser auf (200. Lebenstag). In der Literatur werden zum Geschlechtseinfluß auf die Faserdurchmesser sehr unterschiedliche Angaben gemacht. Augenscheinlich ist, daß aufgrund des dabei verwendeten unterschiedlichen Tiermaterials, der verschiedenen Haltungsbedingungen, Probenentnahmemethoden und Schlachtzeitpunkte eine Vergleichbarkeit der erzielten Ergebnisse unmöglich ist. KNUDSON et al. (1985) finden keine signifikanten Unterschiede bei Vergleichsuntersuchungen zwischen Ebern und Kastraten. Demgegenüber stellten FIEDLER et al. (1995), sowie FIEDLER et al. (1994) fest, daß in den Faserdurchmessern des Kotelettmuskels Unterschiede zwischen Ebern und Börgen auftreten. Eber der Gewichtsklasse $115 \mathrm{~kg}$ weisen bei den untersuchten Genotypen (BHZP, DE x DL, Pi x DL) um 4 - $12 \mu \mathrm{m}$ dickere Fasern auf als Börge. Allerdings konnte bei $95 \mathrm{~kg}$ Lebendmasse noch kein Unterschiede festgestellt werden. Andere Autoren (LARZUL, 1997) diskutieren für Sauen größere Faserdurchmesser, besonders die vom Typ IIBw (fast-twitchnon-oxidative) bei Large White, die mit $100 \mathrm{~kg}$ Körpermasse geschlachtet wurden. Bekanntermaßen unterliegen die IGF-I-Blutplasmakonzentrationen starken Einflüssen durch Geschlecht, Rasse, Fütterung, etc. und hängen auch von der Analysenmethode ab. Dementsprechend ist auch die Schwankungsbreite der Angaben in der Literatur. Vergleichbar mit den von uns ermittelten Werten sind die Angaben von BUONOMO et al. (1987), die bei schnellwüchsigen Rassen höhere IGF-I-Werte fanden, ebenso wie CLUTTER et al. (1995) die bei auf hohe tägliche Zunahmen selektierten Jungsauen Werte um $250 \mathrm{ng} / \mathrm{ml}$ ermitteln konnten. Auch WEILER (1995) bestätigt eine rasse-, geschlechts- und ernährungsabhängige IGF-I-Ausschüttung, die stets mit altersspezifischen Veränderungen des Wachstums einhergehen. ROBERTS et al. (1990) fanden einen signifikanten Einfluß des Geschlechts auf die Plasma-IGF-I-Konzentrationen beim Schaf. GRANT et al. (1991) beobachteten nach Verabreichung von porcinem GH an Schweine eine Erhöhung der Expression der IGF-I mRNA in der Leber, die Expression der IGF-I mRNA in der Skelettmuskulatur jedoch blieb unverändert. Daher kann von einer Wirksamkeit des hauptsächlich in der Leber gebildeten endogenen IGF-I auf das Wachstum der Skelettmuskulatur ausgegangen werden. Die Zusammenhänge zwischen Wachstumsvermögen und IGF-I sind jedoch mit Sicherheit sehr komplex und müssen als Wechselbeziehung verschiedener Hormonsysteme aufgefaßt werden. Dabei spielt auch das Protein Leptin eine Rolle, das als zirkulierendes Signal für den Ernährungsstatus gilt und die Regulation des Körpergewichtes, der Energiebilanz, des Wachstums und der Reproduktion beeinflußt (BLUM, 1997).

Setzt man eine Wechselwirkung zwischen IGF-I und Cortisol für die ausbalancierte Folge von Mitose und Differenzierung beim Schwein voraus, kann Cortisol als das wichtigste Hormon der katabolen Prozesse angesehen werden. Wahrscheinlich ist die Überlegenheit der Rasse Large White in anabolen Eigenschaften hauptsächlich auf deren niedriges Niveau der katabolen Hormone zurückzuführen, d.h. domestizierte Schweine zeigen eine geringere Cortisolsekretion als z.B. Europäische Wildschweine. Das wiederum bestärkt die Hypothese, daß die Selektion auf Wachstumspotential zu einer reduzierten Sekretion von Glucocorticoiden führt. Bei Meishan konnte ein hohes 
Niveau der Gonadensteroide nachgewiesen werden, welches teilweise die niedrigeren IGF-I- und hohen Cortisolwerte zu kompensieren scheint (CLAUS und WEILER, 1996).

Die erwartet positiven Korrelationen zwischen IGF-I-Blutplasmakonzentrationen und bestimmten Wachstumsparametern weisen auf das anabole Potential des IGF-I im Rahmen seiner Wirkung über die GH-IGF-Achse hin. Im eigenen Versuch zeigten Probanden nach der Einteilung in Gruppen anhand des Faserdurchmessers im $M$. triceps brachii signifikante Unterschiede in den IGF-I-Blutplasmakonzentrationen. Da in dieser Gruppe die männlichen Tiere überrepräsentiert sind, scheinen Geschlechtseffekte eine Rolle zu spielen, auch wenn am vorliegenden Material kein geschlechtsspezifischer Unterschied der Muskelfaserdurchmesser an den untersuchten Muskeln ermittelt werden konnte.

\section{Schlußfolgerungen}

- Phänotypisch sichtbare Unterschiede der Bemuskelung der Schultergliedmaße von Ebern und Sauen können ab dem 180. Lebenstag ultrasonographisch bestätigt werden.

- Es können keine signifikanten geschlechtsspezifischen Unterschiede der Muskelfaserdurchmesser $(\mu \mathrm{m})$ des $M$. triceps brachii und des $M$. longissimus dorsi spezifiziert werden.

- Die IGF-I-Blutplasmakonzentrationen der Eber übersteigen am Ende der Mastperiode die Konzentrationen des IGF-I der Sauen und Kastraten deutlich und erreichen etwa den doppelten (Sauen) bzw. dreifachen Wert (Kastraten).

- Tiere mit großen Faserdurchmessern im M. triceps brachii (200. Lebenstag) zeigen signifikant höhere IGF-I-Blutplasmakonzentrationen als Tiere mit niedrigen Faserdurchmessern.

Weiterführende immunhistochemische Untersuchungen am vorliegenden Probenmaterial sollen Aufschluß über die Ausstattung einzelner Muskelzellen und des sie umgebenden Gewebes mit IGF-I, seinen Bindungsproteinen und Rezeptoren geben.

BLUM, W.F.:

\section{Literatur}

Leptin. The voice of the adipose tissue. Horm. Res. 48 (1997), 318-320

BUONOMO, F.C.; LAUTERIO, T.L.; BAILE, C.A.; CAMPION, D.R.:

Determination of insulin-like growth factor 1 (IGF1) and IGF binding protein levels in swine. Domest. Anim. Endocrinol. 4 (1987), 23-31

CLAUS, R; WEILER,U.:

Relationships between IGI-I, cortisol, and osteocalcin in peripheral plasma of growing pigs. Exper. Clin. Endocrinol. \& Diabetes 104 (1996), 344-349

CLUTTER, A.C.; SPICER, L.J.; WOLTMANN, M.D.; GRIMES, R.W.; HAMMOND, J.M; BUCHANAN, D.S.:

Plasma growth hormone, insulin like growth factor 1 and insulin- like growth factor binding proteins in pigs with divergent genetic merit for postweaning average daily gain. J. Anim. Sci. 73 (1995), 17761783 
FIEDLER, I.; HARTUNG, M.; ENDER, K.:

Strukturelle und funktionelle Merkmale der Muskelfasern im Kotelettmuskel von Ebern und Börgen. Aus: Die Ebermast. Schriftenreihe des Bundesministeriums für Ernăhrung, Landwirtschaft und Forsten, Angewandte Wissenschaft Heft 449 (1995), 33-39, Landwirtschaftsverlag 1995

FIEDLER, I.; ENDER, K.; WICKE, M.; LENGERKEN, G. v.:

Relationship between microstructure of muscle tissue and stress sensibility in Landrace pigs. Arch. Tierz., Dummerstorf 36 (1994), 525-538

GRANT, A.L.; HELFERICH, W.G.; KRAMER, S.A.; MERKEL, R.A.; BERGEN, W.G.: Administration of growth hormone to pigs alters the relative amount of insulin-like growth factor I mRNA in liver and skeletal muscle. J. Endocrinol. 130 (1991), 331-338

HONEGGER, A.; HUMBEL, R.E.:

Insulin-like growth factors I and II in fetal and aduit bovine serum. J. Biol. Chem. 261 (1986), 569-575

KNUDSON, B.K.; HOGBERG, M.G.; MERKEL, R.A.; ALLEN, R.E.; MAGEE, W.T.:

Developmental comparison of boars and barrows. I. growth rate, carcass and muscle characteristics, J. Anim. Sci. 61 (1985), 789-796

LARZUL, C.; LEFAUCHER, L.; ECOLAN, P.; GOGUE, J.; TALMANT, A.; SELLIER, P.; LE ROY, P.; MONIN, G.:

Phenotypic and genetic parameters for Longissimus Muscle fiber charakteristics in relation to growth, carcass, and meat quality traits in Large White pigs. J. Anim. Sci. 75 (1997), 3126-3137

LITWACK, G.:

Vitamins and Hormones, Advances in Research and Application. Academic Press, Vol. 48, San Diego 1994

RINDERKNECHT, E.; HUMBEL, R.:

The amino acid sequence of insulin-like growth factor I and its structural homology with proinsulin. J. Biol. Chem. 235 (1978), 2769-2776

ROBERTS, C.A.; MCCUTCHEON, S.N.; BLAIR, H.T.; GLUCKMAN, P.D.; BREIER, B.H.:

Developmemtal pattern of Plasma Insulin-like Growth Factor I concentrations in sheep. Dom. Anim. Endocrinol. 7 (1990) 4, 457-464

SATHER, A.P.; JONES, S.D.M.; JOYAL, S.:

Feedlot performance, carcass composition and pork quality from entire male and female Landrace and WEILER, U.: Large White market-weight pigs. Can. J. Anim. Sci., Ottawa 71 (1991), 29-42

Wachstum und Wachstumsregulation beim Schwein. Univ. Hohenheim, Habil.-Schrift, 1995

Eingegangen: 04.08.1999

Akzeptiert: 01.10.1999

Anschriften der Verfasser

Prof. Dr. Dr. h.c. GERHARD von LENGERKEN, Dr. MICHAEL WICKE,

TA SVEN BIEREDER

Martin-Luther-Universität Halle-Wittenberg

Institut für Tierzucht und Tierhaltung mit Tierklinik

Adam-Kuckhoff-Str. 35

D-06108 Halle/Saale

Dr. WILHELM KANITZ, Dr. FALK SCHNEIDER

Forschungsinstitut für die Biologie landwirtschaftlicher Nutztiere

Fachbereich Fortpflanzungsbiologie

Wilhelm-Stahl-Allee 2

D-18196 Dummerstorf 